\title{
Identification of WISP1 as a novel oncogene in glioblastoma
}

\author{
DI JING ${ }^{1 *}$, QIAN ZHANG ${ }^{2 *}$, HAIMING YU ${ }^{3}$, YAJIE ZHAO ${ }^{4}$ and LIANGFANG SHEN ${ }^{1}$ \\ ${ }^{1}$ Department of Oncology Radiotherapy, Xiangya Hospital of Central South University, Changsha, Hunan 410008; \\ ${ }^{2}$ Teaching and Research Section of Surgery, Xiangnan University Affiliated Hospital, Chenzhou, Hunan 423000; \\ ${ }^{3}$ Department of Critical Care Medicine, Hunan Provincial Peopel's Hospital, Changsha, Hunan 410005; \\ ${ }^{4}$ Department of Nuclear Medicine, Xiangya Hospital, Central South University, Changsha, Hunan 410008, P.R. China
}

Received May 11, 2017; Accepted August 10, 2017

DOI: 10.3892/ijo.2017.4119

\begin{abstract}
Glioblastoma is the most common and aggressive primary brain tumor and has a high mortality in humans. However, mechanisms and factors involved in the progression of glioblastoma remain elusive. WISP1 (WNT1 inducible signaling pathway protein 1), has been suggested to be a critical regulator of cancer development. The aim of this study was to investigate the role of WISP1 in regulating the progression of glioblastoma. Clinicopathological characteristics of glioblastoma were assessed, and higher levels of WISP1 were positively associated with advanced clinical stage and a poor prognosis. Consistently, WISP1 expression was significantly upregulated in glioblastoma tissue and cell lines compared with normal tissue and cells. Additionally, inhibition of WISP1 greatly suppressed cell proliferation, migration, and invasion and promoted apoptosis and cell cycle arrest of glioblastoma cells. Further study indicated that downregulation of WISP1 suppressed cell proliferation associated with the gene expression of c-myc and cyclin D1 and cellular signaling such as through the ERK pathway, while inhibiting epithelialmesenchymal transition and MMP9. Finally, knockdown of WISP1 markedly suppressed in vivo tumor growth and sensitized glioblastoma cells to temozolomide. This study identified WISP1 as an oncogene in glioblastoma and suggests
\end{abstract}

Correspondence to: Professor Liangfang Shen, Department of Oncology Radiotherapy, Xiangya Hospital of Central South University, 87 Xiangya Road, Changsha, Hunan 410008, P.R. China

E-mail: 1fshen2008@163.com

${ }^{*}$ Contributed equally

Abbreviations: WISP1, WNT1 inducible signaling pathway protein 1; IHC, immunohistochemistry; SEM, standard error of the mean; FITC, fluorescein isothiocyanate; PI, propidium iodide; Bax, Bcl-2 associated $\mathrm{X}$ protein; TMZ, temozolomide; CCND1, cyclin D1; MMP9, matrix metallopeptidase 9; ERK, extracellular regulated MAP kinase; MEK, MAP kinase-ERK kinase

Key words: WISP1, glioblastoma, proliferation, invasion, TMZ resistance, ERK that WISP1 may serve as a potential molecular marker and treatment target for glioblastoma.

\section{Introduction}

Gliomas, which comprise $80 \%$ of malignant primary brain tumors (1), are recognized as the leading cause of death from central nervous system tumors (2). Gliomas are generally categorized according to the International Classification of Disease for Oncology (3rd edition) and World Health Organization grading system (3), classifying malignant behavior as grades I-IV. The overall incidence of gliomas is $\sim 6$ per 100,000 persons. The grade IV glioma, glioblastoma (4), accounts for $\sim 45 \%$ of all gliomas and has an overall 5-year survival of $<5 \%$ (1). Despite significant progress in developing surgical and adjuvant therapy, the morbidity and mortality of glioblastoma remain high. In addition to radiotherapy and surgical resection, temozolomide (TMZ) is used as the first-line chemotherapy drug for glioblastoma treatment (5). Use of TMZ has improved the overall survival and progression-free survival, but intrinsic or acquired chemoresistance is major cause of TMZ treatment failure in patients with glioblastoma (6). Identifying novel therapeutic targets is in urgent need for the diagnosis and treatment for glioblastoma.

WNT1 inducible signaling pathway protein1 (WISP1), which belongs to the $\mathrm{CCN}$ family (connective tissue growth factor, cysteine-rich protein, and nephroblastoma overexpressed gene), is a widely recognized downstream target gene of the Wnt/ $\beta$-catenin pathway (7). While members of the CCN family are reported to regulate complex biological processes during embryogenesis, wound healing, and tissue repair (8), WISP1 was originally identified as involved in $\beta$-cateninmediated tumorigenesis (9). WISP1 has been implicated in tumor development in multiple studies. Overexpression of WISP1 is associated with a poor prognosis in colon cancer by regulating cell adhesion (10). WISP1 promotes oral squamous cell carcinoma through angiogenesis (11). In esophageal squamous cell carcinoma, WISP1 regulates tumor cell radiosensitivity (12) and is recognized as a marker suggestive of a poor prognosis (13). These studies strongly suggest that WISP1 plays an important role in tumor development and might therefore be a potential target of tumor therapy.

Aberrant WISP1 levels were recently reported in glioblastoma. Overexpression of WISP1 enhances radiosensitivity of 
glioma cells (14). Hypoxia, an important microenvironment condition for tumor formation, induces WISP1 expression in U87 human glioblastoma cells (15). These observations indicate that WISP1 is an important player in glioblastoma. However, the exact mechanisms by which WISP1 regulates the development of glioblastoma and its potential for clinical therapeutic targeting remain elusive. In the present study, we explored the role of WISP1 in regulating the growth and malignancy of glioblastoma in vitro and in vivo. We also evaluated the potential effect of WISP1 in mediating drug resistance against TMZ in glioblastoma cell lines.

\section{Materials and methods}

Tissue microarray and immunohistochemical staining. A human brain gliocytoma and normal tissue microarray containing a total of 120 samples (\#TC0147; Auragene Bioscience Co., Changsha, China) was established based on 40 cases, including 5 normal samples and 35 tumor samples (2 anaplastic oligodendrogliomas, 24 astrocytomas, and 9 glioblastomas). WISP1 protein levels in the tissue specimens were determined by immunohistochemical staining. Briefly, tissue sections from the human brain tissue microarray were deparaffinized in xylene and rehydrated with decreasing concentrations of ethanol. Endogenous peroxidase activity was quenched by incubating for $15 \mathrm{~min}$ in $3 \%$ hydrogen peroxide. High-temperature antigen retrieval was performed in sodium citrate buffer (\#P019IH; Auragene Bioscience Co.). Tissue sections were then incubated with primary WISP1 antibody (\#18166-1-AP, 1:100 dilution; Proteintech, Wuhan, China) or PCNA (AM3547; Abzoom, Dallas, TX, USA) at $4^{\circ} \mathrm{C}$ overnight, followed by incubation with horseradish peroxidase (HRP)-conjugated secondary antibody (\#SA009; Auragene Bioscience Co.). Finally, the sections were colorized with the DAB substrate kit (\#P013IH; Auragene Bioscience Co.). Slides were counterstained with hematoxylin before dehydration, incubated with xylene, and mounted. The slides were evaluated by two pathologists blinded to the diagnosis for each tissue type. Scores ranged from 0 to 4, with 0 indicating no staining for WISP1 protein, 1 indicating $\leq 10 \%$ staining, 2 indicating 10 to $\leq 25 \%$ staining, 3 indicating 25 to $\leq 75 \%$ staining, and indicating $>75$ to $100 \%$ staining. In analyzing the association of WISP1 levels with clinicopathological features, a score of 0 was categorized as no expression, scores of 1 or 2 as low expression, and scores of 3 or 4 as high expression.

Clinical specimens. Normal human brain and glioma specimens were obtained from patients who underwent brain surgery at the Xiangya Hospital, Central South University from March to June 2016. None of the patients had undergone radiotherapy, chemotherapy, or other anticancer treatment before surgery. The histologic features of all specimens were evaluated by pathologists according to the WHO criteria. Written informed consent was obtained from all patients. Protocols in this study were approved by the Medical Ethics Committee of Xiangya Hospital, Central South University. Analysis of WISP1 expression levels in normal and glioblastoma tissues by bioinformatics analysis in GSE4536 from Oncomine datasets. Statistical analysis of the pathologic stage of glioblastoma patients in WISP1 expression from tissue microarray. Statistical analysis of the pathologic stage of glioblastoma patients in WISP1 expression from TCGA datasets.

Reverse transcription and quantitative real-time polymerase chain reaction. Total RNA of normal brain and glioma tissue samples were isolated using a TRIzol kit (\#R1022; Invitrogen Life Technologies, Carlsbad, CA, USA). Reverse transcription of RNA into cDNA was performed with a reverse transcription kit (\#K1622; Fermentans, Vilnius, Lithuania) according to the manufacturer's instructions. Quantitative real-time polymerase chain reaction (qRT-PCR) was performed with SYBRGreen qPCR Mix (\#P2092; Toyobo, Shiga, Japan) in real-time PCR system (\#ABI 7300; Applied Biosystems). Primers for qPCR are as follows: WISP1 sense, 5'-ACGCAG GGAAGA AGTGTC-3'; antisense, 5'-GAGAAGCCAAGCCCATCA-3'. $\beta$-actin sense, 5'-AGGGGCCGGACTCGTCATACT-3'; antisense, 5'-GGCGGCACCACCATGTACCCT-3'. The $\beta$-actin gene was used as an internal control, and all qRT-PCR reactions were performed in triplicate. Relative mRNA levels of genes were calculated based on the $2^{-\Delta \Delta \mathrm{C}_{\mathrm{T}}}$ method (16).

Cell culture. A normal human astrocyte cell line and human glioma cell lines including SHG-44, U-251, and U-373 MG, were obtained from Auragene Bioscience Co. Cells were cultured in RPMI-1640 medium (\#SH30809.01; Hyclone, Hudson, NH, USA) supplied with $10 \%$ fetal bovine serum (\#900-108; Gemini Bio Products) and $100 \mathrm{U} / \mathrm{ml}$ penicillinstreptomycin. All cells were maintained in humidified cell incubators with $5 \% \mathrm{CO}_{2}$ at $37^{\circ} \mathrm{C}$.

shRNA construction and cell transfection. A human WISP1 shRNA (sh-WISP1) construct was generated by inserting the following targeting sequence into an shRNA vector: 5'-CCGGGCTCCTATCAACCCAAGTATTCAAGAGATACT TGGGTTGATAGGAGCTTTTTTG-3'. A non-specific shRNA sequence was used to construct a control shRNA plasmid (sh-NC). For shRNA transfection, cells were plated at a density of $1 \times 10^{5}$ cells/well in 6 -well plates and cultured for $24 \mathrm{~h}$ to reach $80-90 \%$ confluence. Culture medium was replaced with serum-free medium $4 \mathrm{~h}$ prior to transfection, and $4 \mu \mathrm{g} / \mathrm{well}$ of plasmid DNA was then transfected using Lipofectamine 2000 (\#12566014; Thermo Fisher Scientific, Waltham, MA, USA) reagent according to the manufacturer's instructions. Cells were ready for further experiments after $24 \mathrm{~h}$ of culture in growth medium following transfection.

Western blot analysis. Protein samples from cells with or without transfection were extracted using RIPA lysis buffer (\#P002A; Auragene Bioscience Co.) containing proteinase inhibitors. Sample concentrations were measured with a BCA assay. A total of $30 \mu \mathrm{g}$ of protein from each sample was resolved on $10 \%$ sulfate-polyacrylamide gel and electrophoretically transferred onto a nitrocellulose membrane. Blots were then blocked with $5 \%$ milk and incubated with a primary antibody against the protein of interest in 3\% BSA overnight at $4^{\circ} \mathrm{C}$. After washing, the membranes were incubated with HRP-labeled secondary antibody, and signals were detected using an ECL chemiluminescence kit (\#P001WB-1; Auragene Bioscience Co.). Densitometry analysis was evaluated by IPP6.0 
(Image-Pro Plus v 6.0, Media Cybernetics, Silver Spring, MD, USA). The following antibodies were used: anti-WISP1 antibody (\#18166-1-AP, 1:500; Proteintech), anti-Bax antibody (\#AM2198, 1:800; Abzoom), anti-Bcl-2 antibody (\#YT0496, 1:1,000; ImmunoWay, USA), anti-caspase 3 polyclonal antibody (\#YC0026, 1:1,000; ImmunoWay), anti-c-myc antibody (\#YT0990, 1:1,000; ImmunoWay), anti-CCND1 antibody (\#AM2311, 1:1,000; Abzoom), anti-p53 antibody (\#BM0158, 1:1,000; Abzoom), anti-MMP9 antibody (\#BM0616, 1:1,000; Abzoom), anti-E-cadherin antibody (\#BM0530, 1:1,000; Abzoom), anti-vimentin antibody (\#BM0147, 1:1,000; Abzoom), anti-ERK1/2 antibody (\#YM0224, 1:1,000; ImmunoWay), anti-phospho-ERK1/2 (Y222/205) antibody (\#YP0497, 1:1,000; ImmunoWay), anti-MRK-2 antibody (\#YM0435, 1:1,000; ImmunoWay), anti-phospho-MRK-2 (T394) antibody (\#YP0169, 1:1,000; ImmunoWay), goat anti-rabbit IgG-HRP antibody (\#SA009, 1:15,000; Auragene Bioscience Co.), and goat anti-mouse IgG-HRP antibody (\#SA001, 1:15,000; Auragene Bioscience Co.).

Cell proliferation assay. The effect of WISP1 knockdown on the proliferation of glioma cell lines was determined by a cell proliferation assay. Cells transfected with sh-NC or sh-WISP1 were plated in 96 -well plates at a density of $5 \times 10^{3}$ cells/well with a total volume of $100 \mu \mathrm{l} /$ well. When the cells were ready for the proliferation assay, $10 \mu \mathrm{l}$ of 3-(4,5-dimethylthiazol2-yl) -2,5-diphenyltetrazolium bromide (MTT) solution (\#A600799-0005; Sangon Biotech) was added to each well and the cells were incubated with $5 \% \mathrm{CO}_{2}$ at $37^{\circ} \mathrm{C}$ for an additional $4 \mathrm{~h}$. Formazan formed in each well was solubilized by $150 \mu \mathrm{l}$ DMSO with a $10-$ min gentle shake incubation. The optical density of the solution was measured at $570 \mathrm{~nm}$ (\#MK3; Thermo Fisher Scientific).

Annexin V-FITC/PI staining assay. Human glioma cells with or without WISP1 knockdown were harvested using trypsin that did not contain EDTA. For evaluation of apoptosis, cells were washed with PBS and resuspended in $500 \mu \mathrm{l}$ binding buffer and incubated sequentially with Annexin V-FITC and propidium iodide (PI) according to the manufacturer's instructions using an Annexin V-FITC-PI double staining kit (\#KGA108; KeyGen Biotech, Nangjing, China). Stained cells were evaluated by flow cytometric analysis (\#FACSCanto II; BD Biosciences, Frankin Lakes, NJ, USA). For cell cycle analysis, cells were washed with PBS and fixed in $75 \%$ precooled ethanol overnight at $4^{\circ} \mathrm{C}$. Fixed cells were washed with PBS again and treated with RNase A before staining with PI according to the manufacturer's instructions (\#P8080; Solarbio, Beijing, China). Stained cells were detected by flow cytometric analysis at $488 \mathrm{~nm}$.

Cell migration and invasion assay. The migration and invasion ability of human glioma cell lines were assessed by a Transwell assay. U373-MG and U251 cell lines were cultured or transfected with either sh-NC or sh-WISP1. Cells were then harvested and plated at a density of $1 \times 10^{5}$ cells/well in the upper compartments of Matrigel Transwell chambers (\#354480; Corning) with serum-free medium. For the invasion assay, the upper chambers were precoated with an ECM matrix gel layer. The chambers were inserted into 24 -well plates filled
Table I. Correlation of WISP1 expression and clinicopathological features of glioblastoma patients.

\begin{tabular}{lcccc}
\hline & \multicolumn{3}{c}{ WISP1 expression } \\
\cline { 3 - 3 } $\begin{array}{l}\text { Clinicopathological } \\
\text { features }\end{array}$ & $\begin{array}{c}\text { No. of } \\
\text { cases }\end{array}$ & Low & High & P-value \\
\hline Age (years) & & & & 0.276 \\
$>40$ & 22 & 10 & 12 & \\
$\leq 40$ & 30 & 18 & 12 & \\
Sex & & & & 0.177 \\
Female & 25 & 16 & 9 & \\
Male & 27 & 12 & 15 & \\
Grade & & & & $0.028^{\mathrm{a}}$ \\
I-II & 28 & 19 & 9 & \\
III-IV & 24 & 9 & 15 & \\
\hline
\end{tabular}

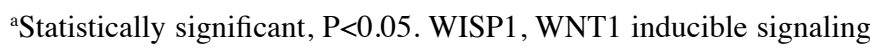
pathway protein 1 .

with cell growth medium (containing $10 \%$ FBS). After incubation for $24 \mathrm{~h}$, cells that had migrated onto the lower membrane were stained with crystal violet solution (\#C0121; Beyotime Biotech) and visualized under a microscope. Further measurement of the optical density at a wavelength of $570 \mathrm{~nm}$ was performed after the cells were treated with glacial acetic acid.

In vivo tumorigenicity assay. To determine tumor growth in vivo, U251 cell lines transfected with Lv-sh-NC and Lv-sh-WISP1 were suspended in culture medium at a density of $1 \times 10^{7}$ cells/ $\mathrm{ml}$. BALB/c mice were acquired at 4 weeks old (certificate no. 43004700016031; Hunan SJA Laboratory Animal Co.) and kept in a 10/14-h light-dark cycle with free access to food and water. Cells were inoculated subcutaneously $\left(4 \times 10^{6}\right.$ cells/injection) into the axillary area of the mice ( 3 mice/group). The growth of the tumors was monitored every 3 days, with mouse bodyweight and tumor size measured and recorded. Any nodule with a diameter $>0.4 \mathrm{~cm}$ was considered to be a tumor. The tumor volume was calculated with the following equation: $\mathrm{V}=\mathrm{A} \times \mathrm{B} 2 / 2 \mathrm{~mm}^{3}$. At the end of experiment, the mice were sacrificed and the tumors were isolated for further measurement.

Statistical analysis. Statistical analysis was performed using SPSS 17.0 statistical software (SPSS, Inc., Chicago, IL, USA). Data are expressed as mean \pm standard error of the mean. $\mathrm{P}<0.05$ was considered to indicate statistical significance. Correlation between WISP1 and clinicopathological features of patients were compared with a $\chi^{2}$ test. Comparisons of WISP1 protein expression, mRNA levels, cell numbers, and tumor parameters were analyzed with a 2-tailed t-test. One-way ANOVA was performed to compare data from more than two groups.

\section{Results}

Upregulation of WISP1 in glioblastoma and its association with clinicopathological features and poor survival. WISP1 

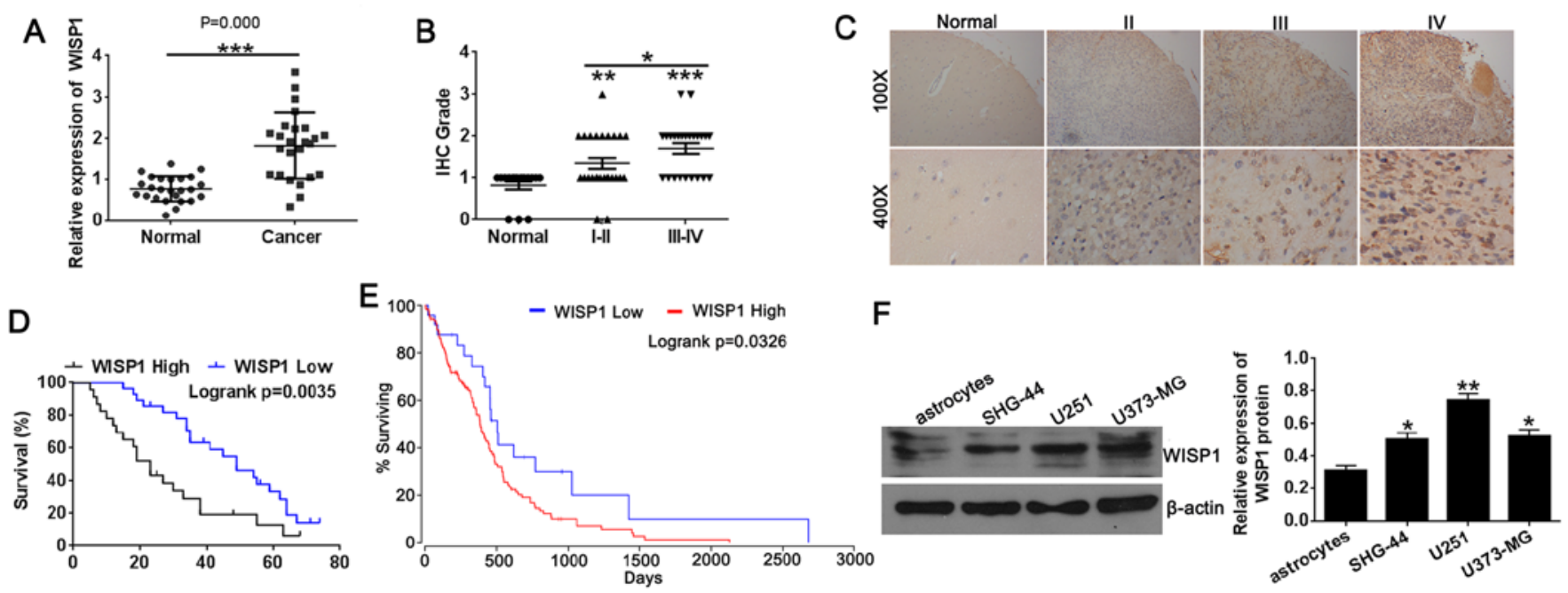

Figure 1. Expression of WISP1 in normal and glioblastoma tissues and cell lines. (A) qRT-PCR assay was used to analyze the expression of WISP1 in 25 pair of normal and glioblastoma tissues. (B) Summary of IHC staining results from tissue microarray sections. $y$-axis represent extent of WISP1 staining according to a 1-4 IHC grading system. $\mathrm{x}$-axis represent the stage of tumors. (C) Representative immunohistochemistry staining of WISP1 protein in tumor sections from different stages of glioblastoma and normal tissues. (D) Statistical analysis on the pathological stage of glioblastoma patients in WISP1 expression from tissue microarray. (E) Statistical analysis on the pathological stage of glioblastoma patients in WISP1 expression from TCGA datasets. (F) Western blot analysis and quantitative analysis of WISP1 protein levels in normal and glioma cell lines. $\beta$-actin protein was used as endogenous loading control. ${ }^{*} \mathrm{P}<0.05,{ }^{* *} \mathrm{P}<0.01$, ${ }^{* * *} \mathrm{P}<0.001$, data represent mean \pm SEM with at least 3 independent experiments.

expression was dramatically higher in glioblastoma tissues than in normal tissues from the Oncomine datasets (GSE4536) (9). We performed qRT-PCR assay and tissue microarray immunohistochemical staining to explore the potential involvement of WISP1 in glioblastoma. The qRT-PCR results and microarray immunohistochemical results showed that WISP1 levels were significantly higher in glioblastoma tissues compared with normal tissues (Fig. 1A and B), similar to the results of the Oncomine datasets (GSE4536). We further analyzed the correlation of WISP1 levels with clinicopathological features of glioblastoma specimens (Table I). The $\chi^{2}$ test indicated that WISP1 expression was positively associated with the clinical stage of glioblastoma $(\mathrm{P}=0.007)$, but no correlation of WISP1 levels with age or sex was detected (all $\mathrm{P}>0.05$ ). As shown in Fig. 1C, the increased expression of WISP1 in normal tissue and in stage II, III, and IV glioblastoma tissues was specifically distributed in the cytosol. In terms of survival, patients with glioblastomas expressing lower levels of WISP1 notably lived longer than those with higher levels of WISP1 expression on tissue microarray (Fig. 1D). We also analyzed data of glioblastoma patients in TCGA datasets, with results similar to those of tissue microarray (Fig. 1E). Those results indicate that WISP1 expression was positively associated with the clinical stage and prognosis in glioblastoma.

We next examined the levels of WISP1 protein in normal astrocytes and glioblastoma cell lines (SHG-44, U251 and U373-MG) by western blotting. Consistent with the tissue results, the protein expression level of WISP1 (Fig. 1F) was significantly higher in glioblastoma cell lines compared with normal astrocytes. These results suggest that higher WISP1 expression was associated with the development of glioblastoma, which correlated with poor survival in patients with glioblastoma.

Downregulation of WISP1 suppresses proliferation and promotes apoptosis and cell cycle arrest of glioblastoma cell lines. Having confirmed that WISP1 is upregulated in glioblastoma cell lines, we hypothesized that high levels of WISP1 are important for maintaining the malignant characteristics of these cells. To test how WISP1 affects glioblastoma cell behavior, we constructed WISP1 shRNA and transfected it into U251 and U373-MG cell lines. As shown in Fig. 2A, western blot results demonstrated efficient knockdown of WISP1 to $30 \%$ of its endogenous levels.

To further assess the role of WISP1 on glioblastoma cell proliferation, MTT assay, and flow cytometry for apoptosis and cell cycle distribution assays were used. Interestingly, knockdown of WISP1 dramatically inhibited cell growth (Fig. 2B and C) as measured by MTT assays. WISP1 deficiency also resulted in increasing cell apoptosis as measured with Annexin V-FITC/PI staining (Fig. 2D). As shown in Fig. 3, transfection of the sh-NC plasimid did not alter cell populations in any phase of the cell cycle compared with non-transfected cells. Both exhibited normal distribution of nearly half the cell population in the G1 phase. However, WISP1 knockdown notably increased cell populations in the G1 phase (by 50\%), with a concomitant decrease of cell populations in the $\mathrm{S}$ phase. These results suggest that downregulation of WISP1 inhibits proliferation of glioblastoma cells by arresting cell cycle progression.

Suppression of WISP1 inhibits migration and invasion of glioblastoma cells. Invasion of surrounding normal tissues is a critical process during tumor progression, so the migratory and invasive capacities of tumor cells are important hallmarks of tumor malignancy. We performed Transwell assays to evaluate the migration of glioma cell lines. Importantly, knockdown of WISP1 greatly inhibited the migratory ability of U251 and U373-MG cells (Fig. 4A), as demonstrated by the $60 \%$ decrease in that cell population that migrated into the lower chambers compared with control groups. Similarly, 
A

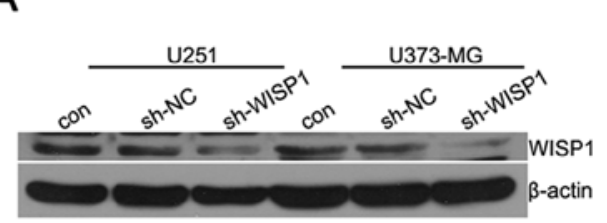

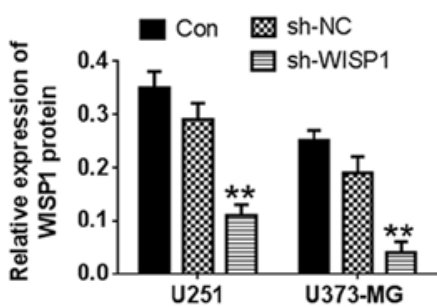

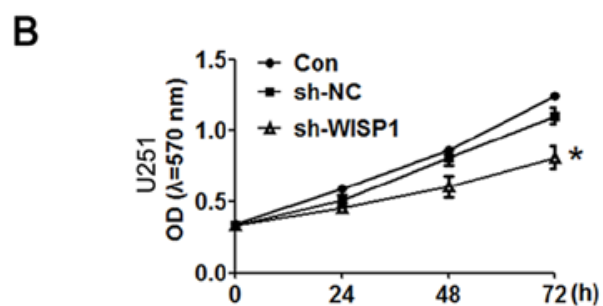

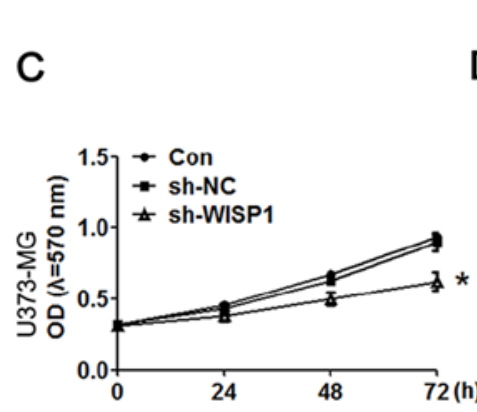
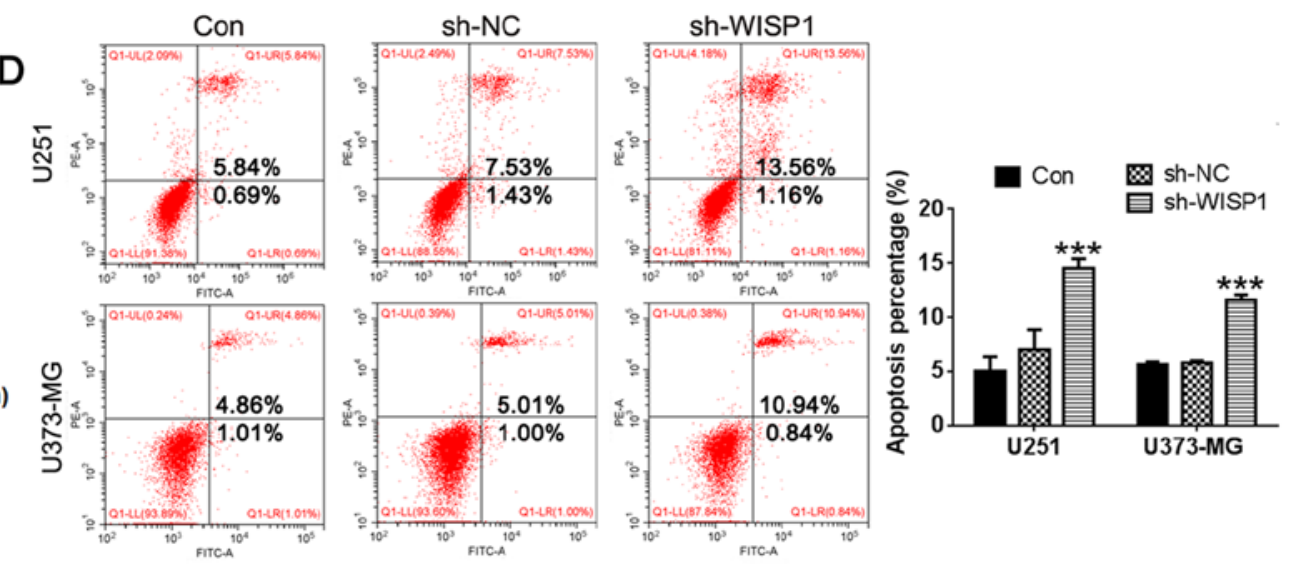

Figure 2. The effect of WISP1 knockdown on the proliferation and apoptosis of glioma cell lines. (A) Western blot analysis of WISP1 protein levels in U251 and U373-MG glioma cell lines that were transfected with control (sh-NC) or sh-WISP1 plasmid. (B and C) MTT assay evaluation of cell proliferation with/ without WISP1 knockdown in U251 (B) and U373-MG (C). Cell growth was monitored every $24 \mathrm{~h}$ for total of $72 \mathrm{~h}$. (D) Flow cytometry detection of apoptosis in glioma cell lines. Annexin V-FITC staining representing cells in early apoptosis stage was shown on x-axis. PE staining representing late apoptosis and necrotic cells is shown on y-axis. Q1 indicates population of necrotic cells, Q2 indicates population of late apoptotic cells, Q3 indicates population of early apoptotic cells, Q4 indicates population of live cells. Quantitative analysis of apoptosis results shown as percentage of apoptotic cells in total cell population. ${ }^{*} \mathrm{P}<0.05,{ }^{* *} \mathrm{P}<0.01,{ }^{* * *} \mathrm{P}<0.001$, data represent mean $\pm \mathrm{SEM}$ with 5 independent experiments.
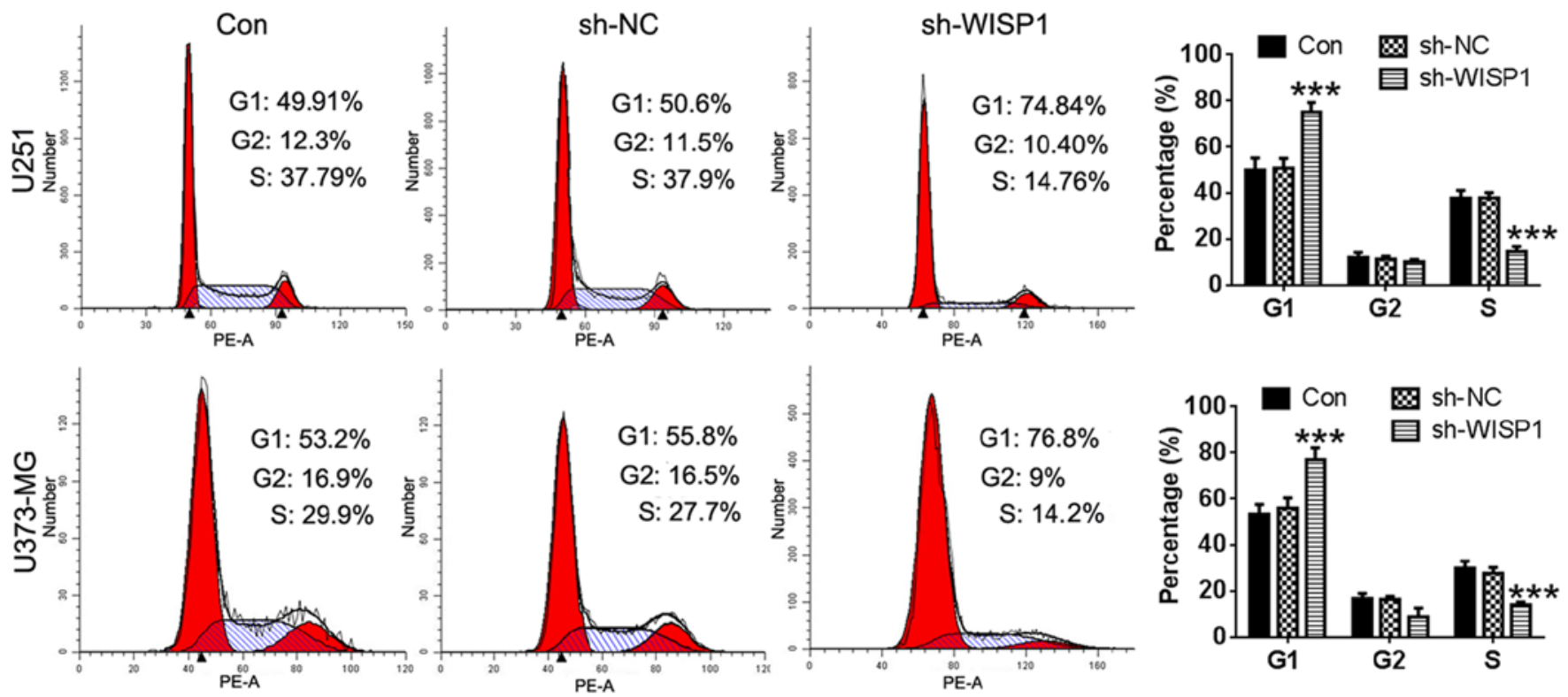

Figure 3. Influence of WISP1 knockdown on cell cycle of glioma cell lines. Left, WISP1 knockdown in U251 and U373-MG glioma cell lines. Flow cytometry was performed to assess the cell cycle status. In each panel, $x$-axis represents the extent of PI staining, which indicate cell DNA content. y-axis represents cell count. Area of the left peak represents cell population in G1 phase, area of the right peak represents cell population in G2 phase, area under the curve in between the 2 peaks represents cell population in S phase. Right, quantitative analysis of left shown as percentage of cells in G1, G2 and S phase of cell cycle comparing to total cell population. ${ }^{* * *} \mathrm{P}<0.001$, data represent mean \pm SEM with at least 3 independent experiments.

WISP1-deficient cells exhibited a 0.5 -fold decrease in invasion through Matrigel to the lower chambers in cell invasion assays(Fig. 4B).
WISP1 downregulation improves drug sensitivity of glioblastoma cells to temozolomide. TMZ is a recognized and effective drug to improve the outcome of glioma, but drug 

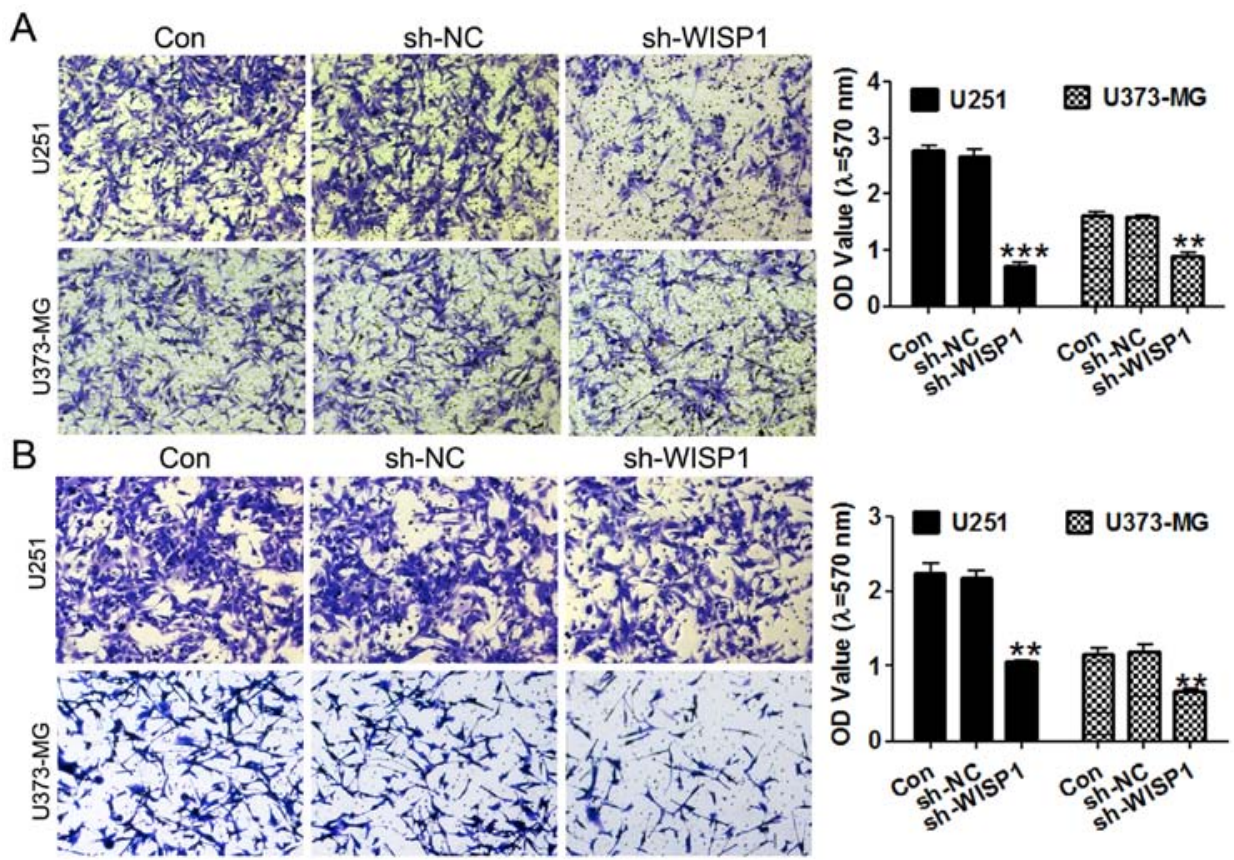

Figure 4. Effect of WISP1 knockdown on migration and invasion of glioma cell lines. WISP1 knockdown in U251 and U373-MG glioma cell lines. (A) Transwell assay evaluation of cell migration and (B) invasion (with pre-coated ECM matrix gel in upper chamber). Images shown are representative observation recording of cell migrated/invaded into bottom chambers which were stained with crystal violet solution. Right panels are quantitative analysis of results from both Transwell experiments. ${ }^{* *} \mathrm{P}<0.01,{ }^{* * *} \mathrm{P}<0.001$, data represent mean $\pm \mathrm{SEM}$ with 3 independent experiments.

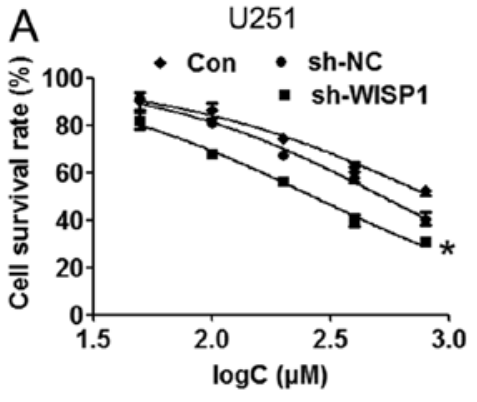

U251

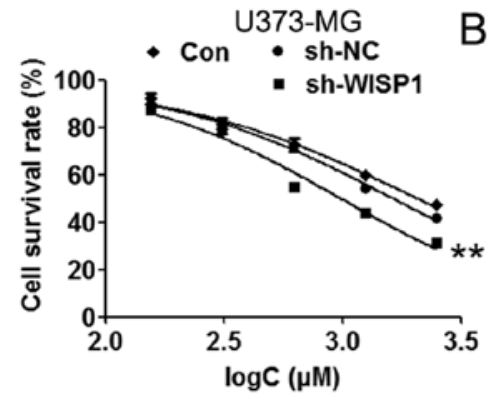

$\log C(\mu \mathrm{M})$
C
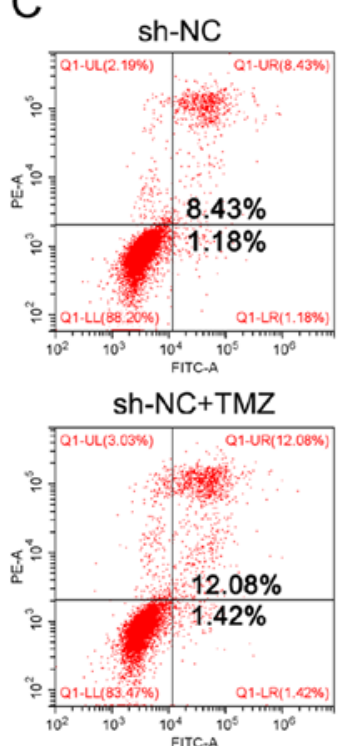
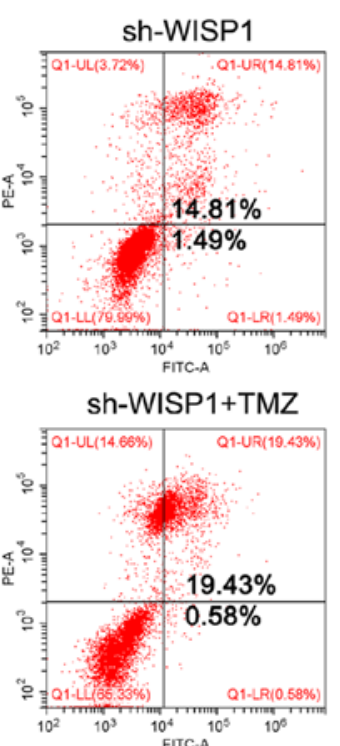

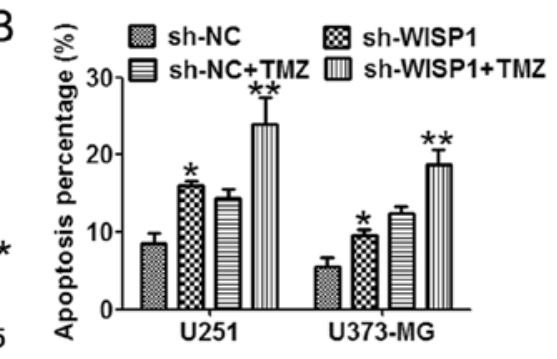

U373-MG

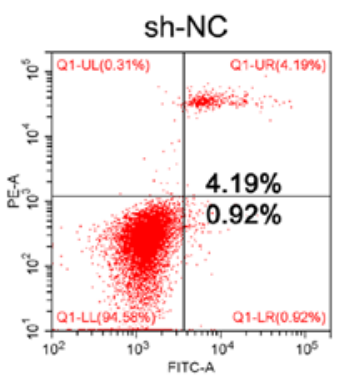

sh-NC+TMZ

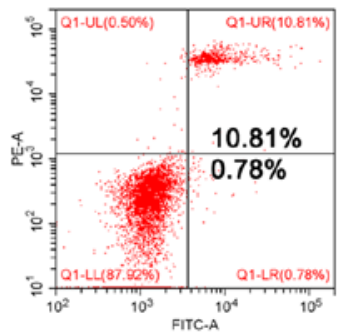

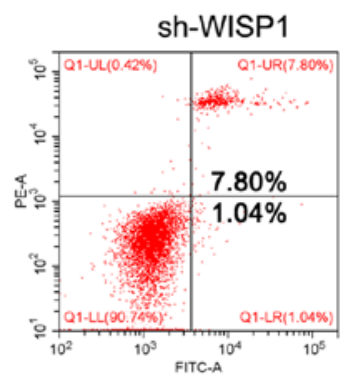

sh-WISP1+TMZ

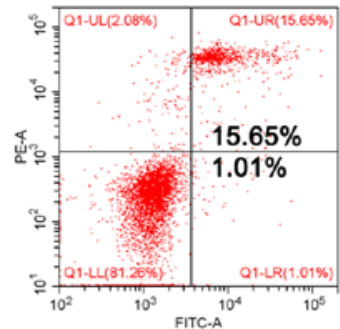

Figure 5. TMZ drug tolerance in WISP1 knockdown glioma cell lines. (A) After knockdown of WISP1, U251 and U373-MG glioma cells were treated with indicated dosages of TMZ for $24 \mathrm{~h}$, MTT assay was performed to evaluate the viability of cells. (C) U251 and U373-MG glioma cells with/without WISP1 knockdown were treated with $500 \mu \mathrm{M} \mathrm{TMZ}$ for $24 \mathrm{~h}$, flow cytometry was used to assess apoptosis of cells. (B) Quantitative analysis of $(\mathrm{C})$. "P<0.05, ${ }^{* *} \mathrm{P}<0.01$, data represent mean \pm SEM with 5 independent experiments. 
A

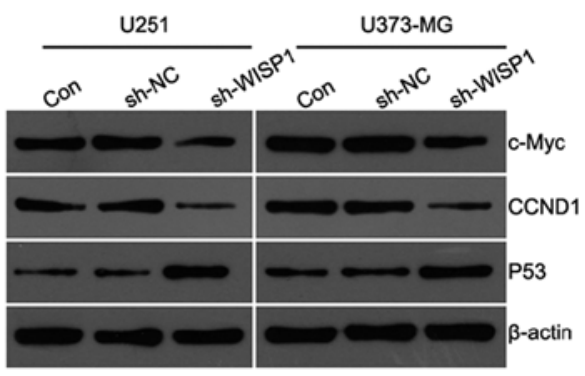

B

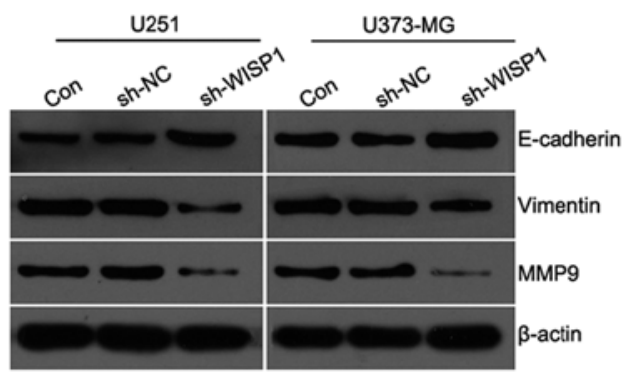

C

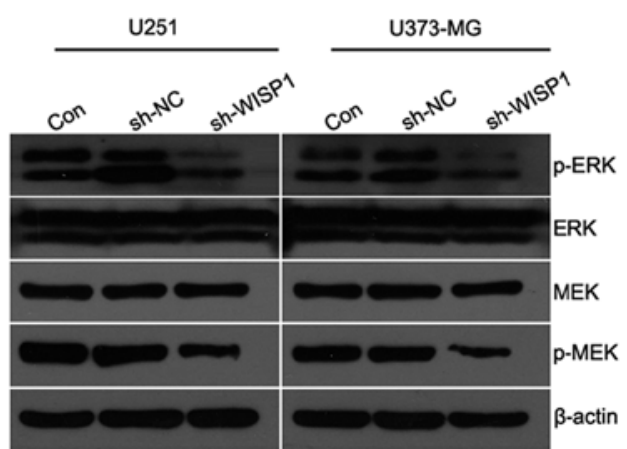

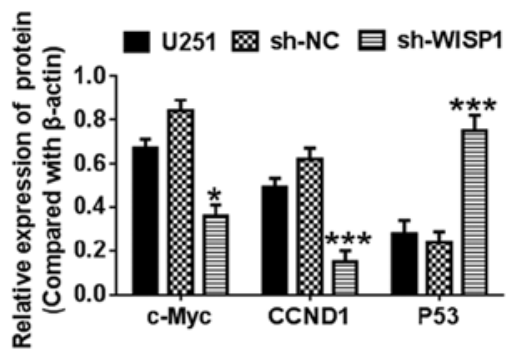
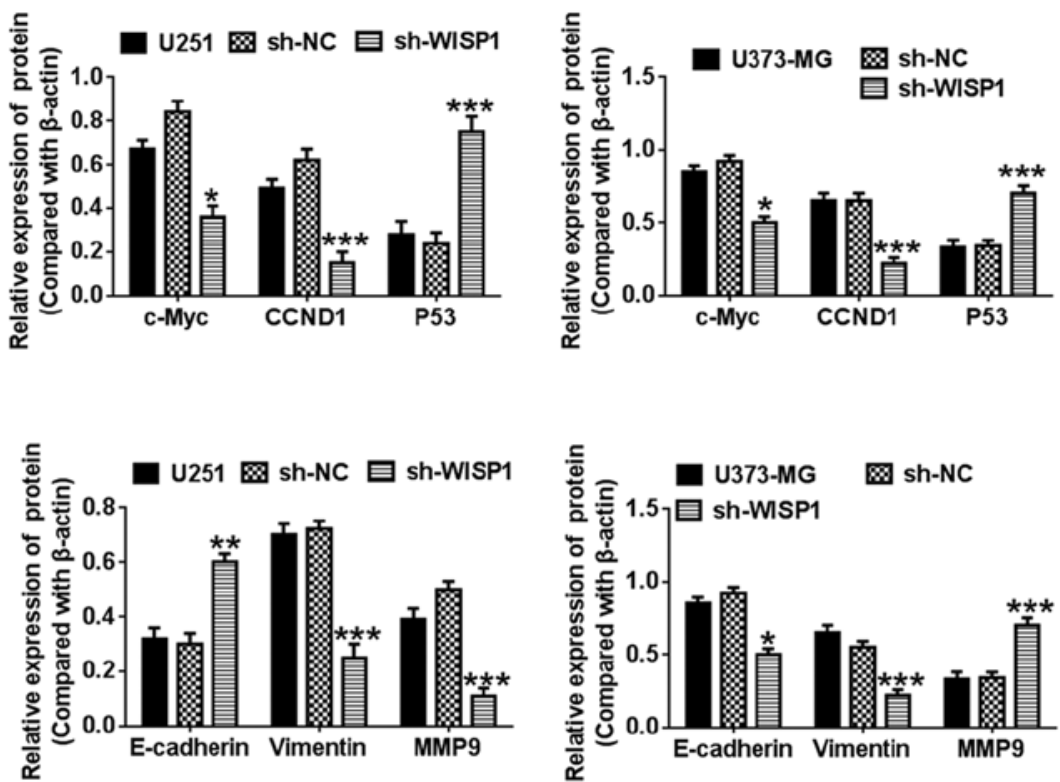

Figure 6. Effect of WISP1 knockdown on signaling pathways associated with proliferation and migration in glioma cell lines. After knockdown of WISP1 in U251 and U373-MG glioma cell lines, protein levels of c-Myc, CCND1, p53 (A), E-cadherin, Vimentin, MMP9 (B), ERK, MEK and phosphorylated form (p-ERK, p-MEK) (C) were evaluated with western blotting. Right panels are quantitative analysis of protein levels that were normalized to $\beta$-actin. ${ }^{*} \mathrm{P}<0.05$, ${ }^{* * *} \mathrm{P}<0.01,{ }^{* * *} \mathrm{P}<0.001$, data represent mean \pm SEM with 5 independent experiments.

resistance often appears to hinder the effectiveness of TMZ. The mechanisms of TMZ resistance in glioma cells are not well understood. In order to study the potential role of WISP1 in regulating glioma drug resistance to TMZ, the cell survival rate and apoptosis were evaluated by MTT assay and flow cytometry in WISP1 downregulated cells with or without TMZ treatment. MTT assay results showed that cells with downregulation of WISP1 had a significantly lower survival rate than control groups, indicating enhanced drug sensitivity to TMZ (Fig. 5A). Flow cytometry results (Fig. 5B and C) demonstrated that WISP1 knockdown was associated with dramatically increased cell apoptosis upon TMZ treatment compared with the control group.

Potential cellular mechanisms of WISP1 function in glioma cells. To further explore the mechanisms by which WISP1 regulates cell proliferation and migration of glioma cells, associated cellular pathways that are responsible for regulating cell growth and tumor malignancy were evaluated by western blotting. Upon WISP1 knockdown, levels of c-myc protein, a key regulator of cell proliferation, greatly decreased. Cyclin D1, an important cell cycle regulation factor, as well as profoundly increased tumor suppressor P53 expression (Fig. 6A). Additionally, epithelial markers of E-cadherin were greatly increased, while the mesenchymal marker vimentin and matrix metallopeptidase 9 (MMP9) level was suppressed (Fig. 6B), indicating that WISP1 may regulate migration of glioma cells by way of epithelial-mesenchymal transition (EMT). Moreover, the extracellular signal-regulated kinase (ERK) signaling pathway, a major regulator of cell proliferation, survival, differentiation, and motility was suppressed by WISP1 knockdown (Fig. 6C). Collectively, these findings indicate that WISP1 may be involved in progression of glioma through regulating classic pathways in cell proliferation, migration, and apoptosis.

Downregulation of WISP1 suppresses glioblastoma tumor growth in vivo. In order to examine the involvement of WISP1 in tumor initiation and progression in vivo, the U251 cell line transfected with control or Lv-sh-WISP1 was evaluated for tumorigenesis capacity in BALB/c-nude mice. Interestingly, tumor volume was significantly smaller in the WISP1 knockdown group (Fig. 7A and B). Consistently, tumor weight was considerably lower in mice with WISP1 

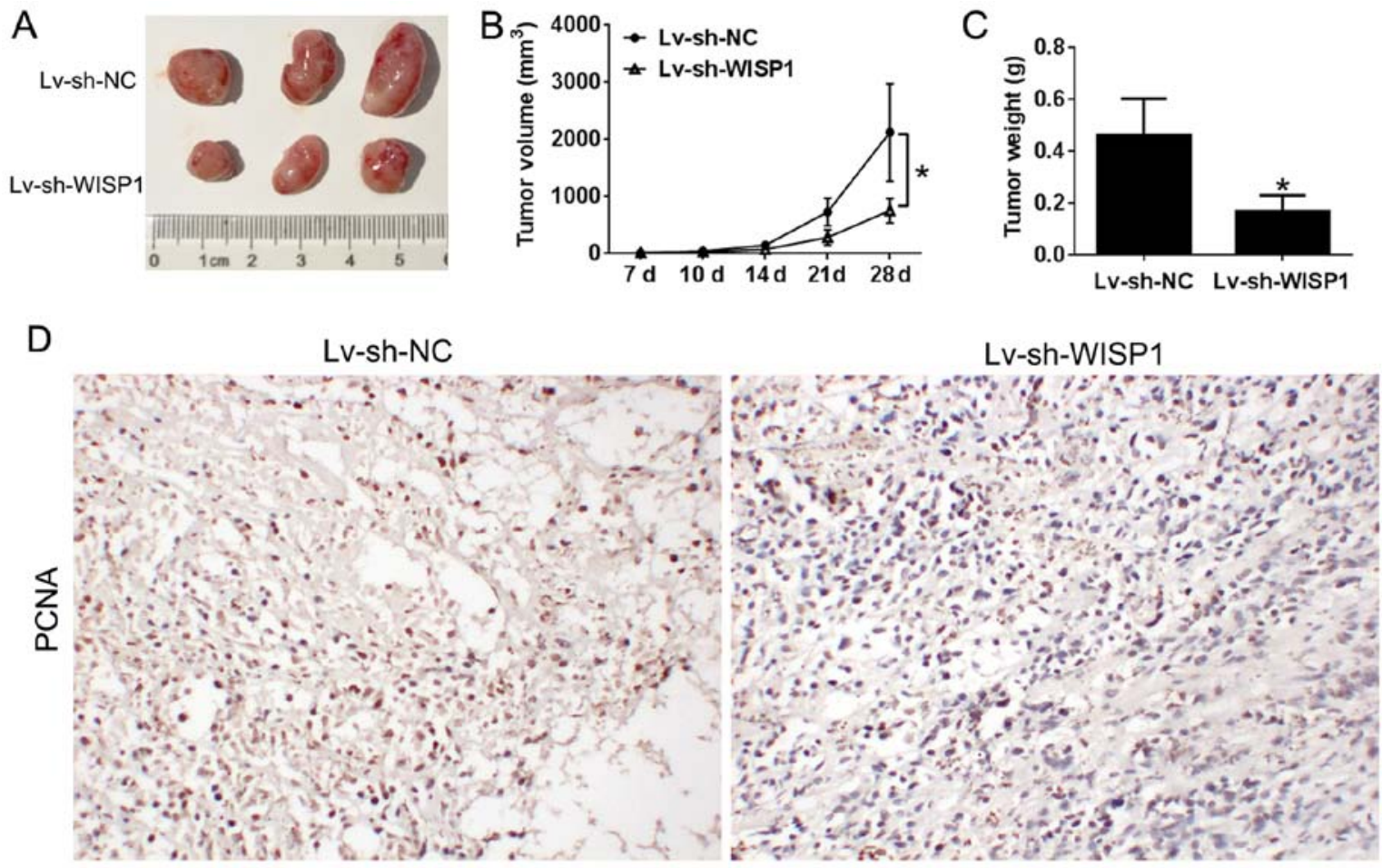

Figure 7. In vivo tumorigenesis ability of glioma cell line upon WISP1 knockdown. U251 cells with/without WISP1 knockdown were inoculated into BALB/c nude mice. (A) Appearance of tumors that were collected at the end of the study. (B) Tumor size was measured across the study period and tumor volume were calculated. (C) Tumor weight were compared between groups at the end of the study. (D) Representative immunohistochemistry staining of PCNA protein in tumor sections from mouse tumor tissues. ${ }^{*} \mathrm{P}<0.05$, data represent mean $\pm \mathrm{SEM}$ with 3 independent experiments.

downregulation(Fig.7C). As detected by immunohistochemical assay, the growth marker PCNA was significantly lower in the WISP1 knockdown group than in the control group (Fig. 7D). These results strongly suggest downregulation of WISP1 in glioblastoma suppresses tumor growth in vivo.

\section{Discussion}

In the present study, we explored the biological functions of WISP1 in glioblastoma. WISP1 levels were markedly increased in glioblastoma compared with normal tissue. Consistently, we found that WISP1 expression was significantly higher in glioblastoma cell lines compared with normal astrocytes. Analysis of clinical characteristics revealed that high WISP1 levels are positively associated with advanced pathologic stage and poor prognosis. Therefore, our study suggests a potential oncogenic role for WISP1 in glioblastoma. This hypothesis is further supported by our subsequent in vitro studies indicating that suppression of WISP1 in glioblastoma cells inhibited proliferation, migration, and invasion and induced apoptosis and cell cycle arrest. Mechanistically, WISP1 may function through regulation of pertinent gene signatures and critical proliferation signaling pathways. Furthermore, WISP1 suppression sensitized glioblastoma cells to TMZ treatment and significantly inhibited tumor growth in vivo.

The initiation and progression of human glioma tumors is a complex, multistep process. Even though a huge number of growth factors, oncogenes, and tumor suppressors have been identified as participating in the regulation of cancer cell survival, proliferation, and invasion, the mechanisms and factors involved in the development of glioblastoma have not been fully elucidated. The aberrant expression of WISP1 has been reported in a number of diseases (17), such as fibrotic diseases, cancer, and diabetic nephropathy and retinopathy. Interestingly, WISP1 is differentially regulated in different types of cancers (colorectal, breast, and esophageal cancer). High levels of WISP1 are associated with poor prognosis in breast (18), rectal (19), and esophageal cancers (20), while in melanoma, lower than normal WISP1 levels were found in patients with a poor outcome (21). Here, we demonstrated for the first time that WISP1 is upregulated in glioblastoma tissues and cell lines, and its expression is associated with advanced clinical stages of glioblastoma and a poor prognosis, supporting the hypothesis that WISP1 is a candidate biomarker for disease progression in glioblastoma.

Further supporting information for a link between WISP1 and glioblastoma progression comes from the in vitro and in vivo experiments in this study. Downregulation of WISP1 markedly inhibited cell proliferation, migration, and invasion, cell cycle progression, and tumorigenesis capacity. These findings are in line with previous reports of a tumor suppression effect with inhibition of WISP1 in prostate and colon cancers $(10,22)$ and provide a plausible explanation for WISP1 regulation of the progression of glioblastoma.

Resistance to apoptosis is a fundamental part of carcinogenesis and is critical for chemotherapeutic drug resistance (23). TMZ is a DNA alkylating agent known to induce cell cycle arrest and eventually to lead to apoptosis (24). The main damage produced by TMZ is through modification of DNA or RNA at the $\mathrm{N}$ and $\mathrm{O}$ sites on guanine and the $\mathrm{N}$ site on adenine by the addition of methyl groups. This process produces incorrect pairing of bases, triggering the intervention of a 
mismatch repair system and can be blocked by base excision repair or the activation of DNA glycosylase and demethylating enzymes (25). In our study, we demonstrated that downregulation of WISP1 improved the sensitivity of glioblastoma cell lines to TMZ treatment, suggesting that knockdown of WISP1 may have affected signaling pathways and gene expression that facilitate TMZ action. This is supported by decreased c-myc and increased p53 expression, since both have been suggested to regulate TMZ sensitivity $(5,26)$. For example, BACH1 promotes TMZ resistance in glioblastoma by antagonizing p53 function (27).

It has been shown that EMT and the MAPK signaling pathway are associated with the progression of several cancers (28-30). MMPs promote tumor cell invasion by degrading extracellular matrix proteins and activating signal transduction cascades that increase lethality (31). EMT is a key factor contributing to cancer metastasis and chemoresistance (32). Zhang et al proved that dysregulation of Fra1 expression by $\mathrm{Wnt} / \beta$-catenin signaling promotes glioma metastasis through EMT (32). MALAT1 decreased the sensitivity of resistant glioma cell lines to TMZ by promoting EMT (33). We observed a decrease in MMP9 and EMT in WISP1 knockdown cells, suggesting that WISP1 may mediate glioblastoma cell migration and invasion through regulation of cell adhesion and EMT. In addition, downregulation of WISP1 improved sensitivity of glioblastoma cells to TMZ, perhaps by suppressing EMT.

ERK signal pathway has been repoted to contribute to cancer proliferation and metastasis. Silencing of AQP5 inhibits cell proliferation, migration, and apoptosis of human glioma cells through regulation of the EGFR/ERK/p38 MAPK signaling pathway (34). Hirudin inhibits cell growth via ERK/MAPK signaling in human gliomas (35). We also detected decreased ERK signaling pathway activity, indicating that WISP1 may promote cancer cell proliferation and survival through this pathway. However, exactly how WISP1 regulates gene expression and signaling pathways that are critical to glioblastoma cell function is still not completely understood.

In conclusion, this study showed for the first time that WISP1 is an important regulator of glioblastoma cell proliferation, apoptosis, migration, invasion, and TMZ drug resistance. WISP1 mediates these biological processes through regulating critical gene expression and pathways that are involved in cancer progression. Based on the association of WISP1 with clinicopathological features, downregulation of WISP1 may serve as a therapeutic strategy for glioblastoma.

\section{Acknowledgements}

This study was supported by the Scientific Research Fund of Hunan ProvincialEducation Department (no. 15B225),Scientific Research Fund of Xiangnan University (no. 2015XB12) and Scientific Research Fund of Hunan Provincial Health and Family Planning Commission (no. C2017014).

\section{References}

1. Ostrom QT, Bauchet L, Davis FG, Deltour I, Fisher JL, Langer CE, Pekmezci M, Schwartzbaum JA, Turner MC, Walsh KM, et al: The epidemiology of glioma in adults: A 'state of the science' review. Neuro-oncol 16: 896-913, 2014.
2. Rao JS: Molecular mechanisms of glioma invasiveness: The role of proteases. Nat Rev Cancer 3: 489-501, 2003.

3. Gupta K and Salunke P: Molecular markers of glioma: An update on recent progress and perspectives. J Cancer Res Clin Oncol 138: 1971-1981, 2012.

4. Larjavaara S, Mäntylä R, Salminen T, Haapasalo H, Raitanen J, Jääskeläinen J and Auvinen A: Incidence of gliomas by anatomic location. Neuro-oncol 9: 319-325, 2007.

5. Martin S, Janouskova $\mathrm{H}$ and Dontenwill $\mathrm{M}$ : Integrins and $\mathrm{p} 53$ pathways in glioblastoma resistance to temozolomide. Front Oncol 2: 157, 2012.

6. Pegg AE: Repair of $\mathrm{O}(6)$-alkylguanine by alkyltransferases. Mutat Res 462: 83-100, 2000.

7. Kleer CG: Dual roles of CCN proteins in breast cancer progression. J Cell Commun Signal 10: 217-222, 2016.

8. Holbourn KP, Acharya KR and Perbal B: The CCN family of proteins: Structure-function relationships. Trends Biochem Sci 33: 461-473, 2008.

9. Gurbuz I and Chiquet-Ehrismann R: CCN4/WISP1 (WNT1 inducible signaling pathway protein 1): A focus on its role in cancer. Int J Biochem Cell Biol 62: 142-146, 2015.

10. Wu J, Long Z, Cai H, Du C, Liu X, Yu S and Wang Y: High expression of WISP1 in colon cancer is associated with apoptosis, invasion and poor prognosis. Oncotarget 7: 49834-49847, 2016.

11. Chuang JY, Chen PC, Tsao CW, Chang AC, Lein MY, Lin CC, Wang SW, Lin CW and Tang CH: WISP-1 a novel angiogenic regulator of the $\mathrm{CCN}$ family promotes oral squamous cell carcinoma angiogenesis through VEGF-A expression. Oncotarget 6: 4239-4252, 2015

12. Zhang H, Luo H, Hu Z, Peng J, Jiang Z, Song T, Wu B, Yue J, Zhou R, Xie R, et al: Targeting WISP1 to sensitize esophageal squamous cell carcinoma to irradiation. Oncotarget 6: 6218-6234, 2015.

13. Zhang H, Luo H, Jiang Z, Yue J, Hou Q, Xie R and Wu S: Fractionated irradiation-induced EMT-like phenotype conferred radioresistance in esophageal squamous cell carcinoma. J Radiat Res (Tokyo) 57: 370-380, 2016.

14. Yu W, Song T, Shi L, Wang X, Jiang Z, Zhang $\mathrm{H}$ and Wu S: Targeted inhibition of WISP1 enhanced radiosensitivity in glioma cells. Zhonghua Yi Xue Za Zhi 94: 1507-1511, 2014 (In Chinese)

15. Minchenko OH,Kharkova AP, Minchenko DO and Karbovskyi LL: Effect of hypoxia on the expression of genes that encode some IGFBP and CCN proteins in U87 glioma cells depends on IRE1 signaling. Ukr Biochem J 87: 52-63, 2015.

16. Livak KJ and Schmittgen TD: Analysis of relative gene expression data using real-time quantitative PCR and the 2 (-Delta Delta C (T)) method. Methods 25: 402-408, 2001.

17. Jun JI and Lau LF: Taking aim at the extracellular matrix: CCN proteins as emerging therapeutic targets. Nat Rev Drug Discov 10: 945-963, 2011.

18. Xie D, Nakachi K, Wang H, Elashoff R and Koeffler HP: Elevated levels of connective tissue growth factor, WISP-1, and CYR61 in primary breast cancers associated with more advanced features. Cancer Res 61: 8917-8923, 2001.

19. Tian C, Zhou ZG, Meng WJ, Sun XF, Yu YY, Li L, Luo HZ, Yang L, Zhou B and Gu J: Overexpression of connective tissue growth factor WISP-1 in Chinese primary rectal cancer patients. World J Gastroenterol 13: 3878-3882, 2007.

20. Nagai Y, Watanabe M, Ishikawa S, Karashima R, Kurashige J, Iwagami S, Iwatsuki M, Baba Y, Imamura Y, Hayashi N, et al: Clinical significance of Wnt-induced secreted protein-1 (WISP-1/ CCN4) in esophageal squamous cell carcinoma. Anticancer Res 31: 991-997, 2011.

21. Shao H, Cai L, Grichnik JM, Livingstone AS, Velazquez OC and Liu ZJ: Activation of Notch1 signaling in stromal fibroblasts inhibits melanoma growth by upregulating WISP-1. Oncogene 30: 4316-4326, 2011.

22. Ono M, Inkson CA, Sonn R, Kilts TM, de Castro LF, Maeda A, Fisher LW, Robey PG, Berendsen AD, Li L, et al: WISP1/CCN4: A potential target for inhibiting prostate cancer growth and spread to bone. PLoS One 8: e71709, 2013.

23. De Salvo M, Maresca G, D'agnano I, Marchese R, Stigliano A, Gagliassi R, Brunetti E, Raza GH, De Paula U and Bucci B: Temozolomide induced c-Myc-mediated apoptosis via Akt signalling in MGMT expressing glioblastoma cells. Int J Radiat Biol 87: 518-533, 2011.

24. Nagasawa DT, Chow F, Yew A, Kim W, Cremer N and Yang I: Temozolomide and other potential agents for the treatment of glioblastoma multiforme. Neurosurg Clin N Am 23: 307-322, ix, 2012. 
25. Stavrovskaya AA, Shushanov SS and Rybalkina EY: Problems of glioblastoma multiforme drug resistance. Biochemistry (Mosc) 81: 91-100, 2016.

26. Luo H, Chen Z, Wang S, Zhang R, Qiu W, Zhao L, Peng C, Xu R, Chen W, Wang HW, et al: c-Myc-miR-29c-REV3L signalling pathway drives the acquisition of temozolomide resistance in glioblastoma. Brain 138: 3654-3672, 2015.

27. Nie E, Jin X, Wu W, Yu T, Zhou X, Zhi T, Shi Z, Zhang J, Liu N and You Y: BACH1 promotes temozolomide resistance in glioblastoma through antagonizing the function of p53. Sci Rep 6: 39743, 2016

28. Li DM and Feng YM: Signaling mechanism of cell adhesion molecules in breast cancer metastasis: Potential therapeutic targets. Breast Cancer Res Treat 128: 7-21, 2011.

29. Huan J, Wang L, Xing L, Qin X, Feng L, Pan X and Zhu L: Insights into significant pathways and gene interaction networks underlying breast cancer cell line MCF-7 treated with 17ß-estradiol (E2). Gene 533: 346-355, 2014.

30. Burotto M, Chiou VL, Lee JM and Kohn EC: The MAPK pathway across different malignancies: A new perspective. Cancer 120: 3446-3456, 2014.
31. Choe G, Park JK, Jouben-Steele L, Kremen TJ, Liau LM, Vinters HV, Cloughesy TF and Mischel PS: Active matrix metalloproteinase 9 expression is associated with primary glioblastoma subtype. Clin Cancer Res 8: 2894-2901, 2002.

32. Zhang L, Liu H, Mu X, Cui J and Peng Z: Dysregulation of Fra1 expression by $\mathrm{Wnt} / \beta$-catenin signalling promotes glioma aggressiveness through epithelial-mesenchymal transition. Biosci Rep 37: 37, 2017.

33. Li H, Yuan X, Yan D, Li D, Guan F, Dong Y, Wang H, Liu X and Yang B: Long non-coding RNA MALAT1 decreases the sensitivity of resistant glioblastoma cell lines to temozolomide. Cell Physiol Biochem 42: 1192-1201, 2017.

34. Yang J,Zhang JN, Chen WL, Wang GS, Mao Q, LiSQ, Xiong WH, Lin YY, Ge JW, Li XX, et al: Effects of AQP5 gene silencing on proliferation, migration and apoptosis of human glioma cells through regulating EGFR/ERK/ p38 MAPK signaling pathway. Oncotarget 8: 38444-38455, 2017.

35. Zhao L: Hirudin inhibits cell growth via ERK/MAPK signaling in human glioma. Int J Clin Exp Med 8: 20983-20987, 2015. 